\section{A capillary discharge tube for the production of intense vuv resonance radiation}

\author{
G Schönhense and U Heinzmann \\ Fritz-Haber-Institut der MPG, Faradayweg 4-6, \\ D-1000 Berlin 33, Germany
}

Received 27 May 1982

\begin{abstract}
Design and operational characteristics of a line source with very high intensity in the vacuum uv are described. The source generates rare-gas resonance radiation in a lowpressure, cold-cathode, DC glow discharge up to $200 \mathrm{~mA}$. The windowless lamp is differentially pumped and can be installed inside a vacuum chamber. During operation it is water cooled and can be mildly baked because it contains metal seals only. In a light beam of $2.5 \mathrm{~mm}$ diameter the photon flux for the strongest $\mathrm{HeI}$ and $\mathrm{NeI}$ emission line has been measured to be nearly $10^{13} \mathrm{~s}^{-1}$ and one order of magnitude less for the strongest HeII, NeII and ArI lines. The photon intensities obtained are compared with values from the literature. The lamp has been used to investigate atomic and molecular photoionisation simultaneously resolved with respect to photon and electron energy, electron emission angle, and spin. To show the performance, angular-distribution results of photoelectron intensities for nitrogen molecules $\left(\mathrm{X}^{2} \Sigma_{\mathrm{g}}^{+}, \mathrm{A}^{2} \Pi_{u}, \mathrm{~B}^{2} \Sigma_{u^{+}}\right)$at $58.43 \mathrm{~nm}$ are presented.
\end{abstract}

\section{Introduction}

During the last decade synchrotrons and storage rings became the most promising source of vacuum ultraviolet (vuv) radiation, mainly because of their unique continuous spectral distribution. Nevertheless there are still types of photoionisation experiments which, at the present, can only be carried out using resonance line radiation emitted from electrical discharges in gases, the only intense vuv source for a long time. The reason is that very high light intensities are obtainable at some resonance wavelengths. In angle-resolved photoelectron spin-polarisation spectroscopy experimental success depends most sensitively upon the vUv radiation intensity. For experiments with Ar target atoms (Heinzmann et al 1980), for example, a photon flux of more than $10^{11} \mathrm{~s}^{-1}$ (with a bandwidth less than $0.1 \mathrm{eV}$ in order to resolve the ionic fine-structure splitting of $0.18 \mathrm{eV}$ ) is essential to yield a detectable photoelectron signal in the spin analyser. Only very recently photon fluxes of this order of magnitude could be achieved (Wuilleumier 1981) from a storage ring using a special high-flux monochromator under optimum operating conditions. It is the purpose of this paper to present a discharge lamp which meets the intensity requirements mentioned above at twelve discrete wavelengths of $\mathrm{He}, \mathrm{Ne}$ and $\mathrm{Ar}$ resonance lines between $30 \mathrm{~nm}$ and $110 \mathrm{~nm}$.

The types of line sources most widely used for ultraviolet photoelectron spectroscopy are:

(i) the DC cold-cathode discharge (Samson 1967), modifications of which are the hollow cathode (Newburgh et al 1962) and the charged particle oscillator (Burger and Maier 1979);

(ii) the duoplasmatron (Samson and Liebl 1962), which is a DC low-pressure arc in a magnetic field;

(iii) the cavity microwave discharge (Fehsenfeld et al 1965), which can be optimised by applying an external magnetic field (Vorburger et al 1976).

Among the different types we selected the cold-cathode discharge tube because its construction is easy and reliable, and it can be built very small, thus allowing a close distance between source and target. Consequently a relatively large solid angle interval of emitted radiation can be used at the target, the diameter of which is restricted to a few $\mathrm{mm}$ in angle-resolved photoelectron spectroscopy. The advantages of a small design were rated higher than the special advantages of other constructions: the extremely low discharge pressure (down to some $10^{-4} \mathrm{~Pa}$ ) of the charged particle oscillator, causing high intensity ratios between ionic and atomic lines; the high discharge current (several amps) of the duoplasmatron, yielding high plasma densities; or the electrodeless construction of the cavity microwave discharge source, which makes it easy to get clean discharge conditions.

Absolute photon fluxes achieved with the different types of line sources are compared in $\S 2.3$. The resonance lamp described in the following has been used routinely for the past three years in the investigation of angular distribution and spin polarisation of photoelectrons from permanent gases (Heinzmann 1980, and references therein) and metal vapours (Schönhense et al 1982a and b). During an overall time of operation of more than 1000 hours not a single malfunction of the lamp occurred.

\section{Specifications of the lamp}

Photoionisation of most of the gaseous targets requires ionising radiation of wavelengths shorter than about $100 \mathrm{~nm}$. Below the $\mathrm{LiF}$ cut off at $104 \mathrm{~nm}$ no transparent bulk material for windows or optical elements exists. Just very thin metal foils or films of some organic compounds transmit some percents of this radiation and almost all gases are opaque due to their high photoabsorption cross sections. Hence this region is commonly called the vacuum uv.

The discharge needs a working pressure of some $10^{2} \mathrm{~Pa}$, whereas the vacuum in the main chamber must be in the $10^{-4} \mathrm{~Pa}$ range with the lamp operating. To maintain the pressure difference, either a thin window or one or more stages of differential pumping have to be used. We chose a differentially-pumped, windowless light source. It seemed to be advantageous in gas-phase photoionisation, because the pressure rise of $10^{-4} \mathrm{~Pa}$ in the vacuum chamber due to gas leakage from the windowless lamp can be accepted. vuv windows reduce the photon intensity by one order of magnitude or more depending upon the wavelength (Samson 1967) and their transmission tends to decrease with increasing time of lamp operation caused by contamination of the window surfaces. In addition, the very thin VUV windows are extremely sensitive to pressure differences and thus not easy to handle.

It is an experimental experience (Kinsinger et al 1972. Fellner-Feldegg et al 1974) that gas discharges have to be operated under very clean conditions if high photon fluxes are desired. This important aspect has been taken into account concerning construction and operation of the lamp described.

\subsection{Constructional details}

Figure 1 shows a longitudinal section of the discharge lamp.

(i) The lamp body contains discharge capillary, anode and a part of the light capillary. It has two flanges to fasten the cathode and the differential pumping stage. It is machined from copper and a water-cooling coil is soldered onto its outer surface to carry off the thermal energy produced in the discharge. The flanges have two radial bores for the connection with the adjustable mounting.

(ii) The discharge capillary of aluminium oxide $\left(\mathrm{Al}_{2} \mathrm{O}_{3}\right)$ 


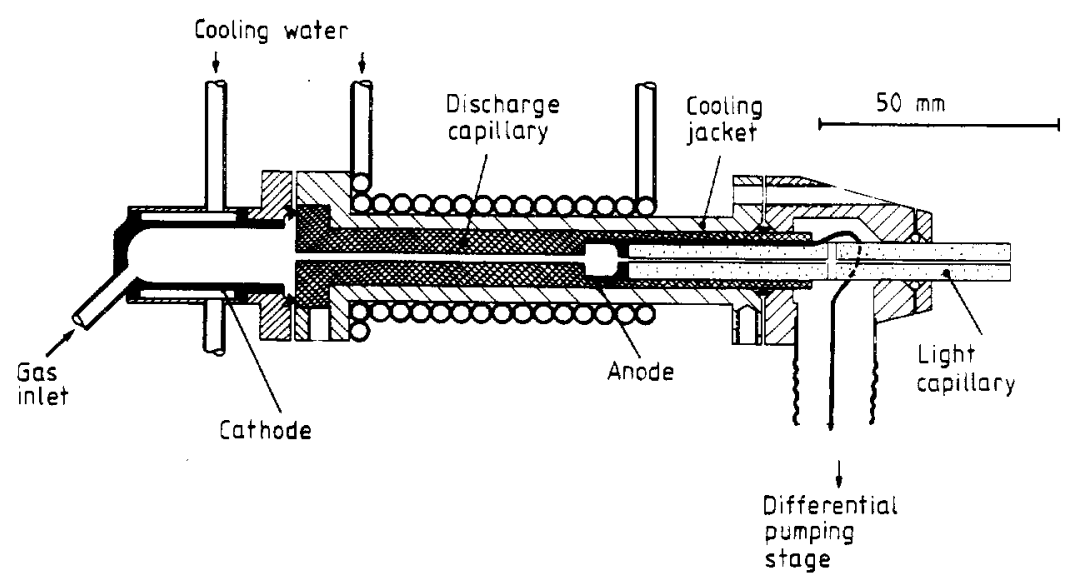

Figure 1. Scaled longitudinal section of the vuv light source (version with one differential pumping stage).

ceramics is machined to high precision witn diamond grinding tools and fits into the copper cooling jacket. Its inner diameter is $2 \mathrm{~mm}$ with a length of $60 \mathrm{~mm}$. The capillary carries a flange with a plane face to allow metal-sealed connection with the cathode. It is a special feature of the lamp that anode and light capillary are inserted into the discharge tube, so that alignment is easy and there is no high-voltage electrode on the outside of the lamp body. The walls of the discharge tube have to abduct the thermal energy from the anode. Because of its relatively high thermal conductivity, high-purity (99.7\%) $\mathrm{Al}_{2} \mathrm{O}_{3}$ ceramics was chosen as capillary material instead of the more usual quartz capillary (e.g. Brundle et al 1974, Poole et al 1974, Ueno et al 1977, also commercial lamps).

(iii) The cathode consists of a water-cooled hollow cylinder made from a copper-beryllium bronze (as described below) which is soldered into a commercial miniconflat (CF16) flange. At the end of the cathode the discharge gas enters the lamp via a thin stainless-steel tube. The cathode is sealed against the discharge capillary by means of a soft aluminium gasket ( $99.9999 \%$ purity) with a triangular profile pressed directly onto the ceramic face. This technique provides a very simple, reliable, bakeable and removeable high-vacuum sealing between metal and ceramics. For the present purpose the great advantage of this connection compared to soldered metal-ceramics sealings (e.g. Neddermeyer et al 1976) is that it can be easily demounted. This becomes necessary after approximately every 200 hours of operation, because metallic deposits of sputtered cathode material on the inner walls of the discharge capillary diminish the light intensity. The deposits can be removed by etching the whole $\mathrm{Al}_{2} \mathrm{O}_{3}$ tube in an acid bath for some hours.

The cathode is exposed to a continuous ion bombardment which causes electrode erosion or sputtering. To find out an electrode material with a good resistance against sputtering, cathodes of four different metals were tested over equal operating periods under comparable conditions. Pure aluminium (99.9999\%) was expected to be almost free from sputtering (Rosebury 1965), and has been used in most of the discharge lamps described in the literature (e.g. Newburgh et al 1962, Poole et al 1974, Fellner-Feldegg et al 1974, Bruck et al 1976, Katsumata et al 1979b). But it turned out to show a very rough cathode surface and the discharge capillary was markedly metallised after some 30 hours of continuous lamp operation with a discharge current of $150 \mathrm{~mA}$. Duralumin (used by Ueno et al 1977) appeared to be slightly more resistant but the results were not satisfying, either. Stainless-steel cathodes (e.g. Neddermeyer et al 1976, Brundle et al 1974) had apparently better erosion properties but, caused by thermal stress, the inner surface of the material became ferromagnetic. This can be a source of errors in angular-resolved measurements with slow electrons if the lamp is installed very close to the target. An alloy of copper $(87 \%)$, cobalt ( $12.5 \%)$ and beryllium $(0.5 \%)$ ("Beryvac $60^{\prime}$ of Vacuumschmelze $\mathrm{GmbH}$ ) was found to be the best suited cathode material of all four metals tested. The cathode surface remained smooth and only the face of the capillary was slightly metallised. It should be mentioned that the cathode area of the present lamp is relatively small because of geometrical restrictions. A larger cathode would certainly reduce the sputtering rate.

(iv) The anode is machined of the same $\mathrm{Cu}-\mathrm{Co}-\mathrm{Be}$ bronze as used for the cathode. A teflon-insulated wire from the anode to the power supply runs through a groove in the light capillary and inside of the differential pumping port. The electrical feedthrough is at the end of the flexible pumping tube, not shown in figure 1. Using this technique of electrical connection the lamp is electrically neutral, which is advantageous if it is placed in the vacuum chamber close to the target. In contrast to the ion bombardment on the cathode, the electrode erosion caused by the electron impact is very weak only, so the anode area can be kept small.

(v) The light capillary is inserted into the enlarged end of the discharge tube so that its bore is automatically aligned with the inner bore of the discharge capillary. It is a commercial Pyrex glass capillary which enables the radiation to emerge into the high-vacuum chamber without passing through a window. Differential pumping is made possible across a transverse bore. The light capillary can easily be changed by loosening the small front flange of the lamp. Capillaries with inner diameters from $0.8 \mathrm{~mm}$ to $2 \mathrm{~mm}$ have been used (for differences in the corresponding photon fluxes, see $\S 2.3$ ).

(vi) Differential pumping is employed in order to reduce the leakage of discharge gas into the high-vacuum chamber. It is necessary because the pressure in the discharge capillary lies between 10 and several $100 \mathrm{~Pa}$ (depending on the resonance line produced), whereas the high-vacuum is some $10^{-4} \mathrm{~Pa}$. The pumping stage consists of a stainless-steel lathe work with end flanges and a flexible pumping tube. It is sealed against discharge tube and light capillary by means of aluminium gaskets with triangular profile. Also $\mathbf{O}$ ring seals of viton could be used in case the lamp was only mildly baked $\left(100^{\circ} \mathrm{C}\right)$. In experiments using a plane polariser (Schönhense 1980) the lamp has been operated with two stages of differential pumping. The second stage was built similar to the first one; the position of the 


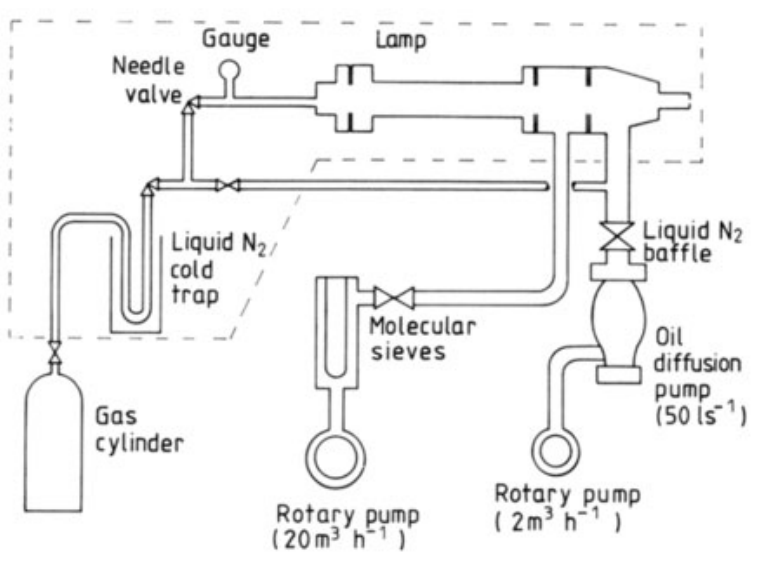

Figure 2. Schematic diagram of the discharge-gas supply and pumping system. The broken line shows the baking zone.

second transverse bore in the light capillary corresponds to the end of the light capillary in the one-stage version (figure 1).

(vii) The gas supply and pumping sy'stem is shown in figure 2. The discharge gas (helium $99.9999 \%$, neon $99.995 \%$ or argon 99.999\%) from a commercial steel cylinder (Messer Griesheim) is purified from water or oil contaminations in the tubes by flowing through a liquid-nitrogen cold trap. The inlet system consists of bakeable UHV components only, in order to have very clean conditions for the discharge. The gas enters the lamp through a leak valve. The gas pressure is measured just behind the valve using a bakeable heat-conduction gauge. After passing the discharge region the main part of the gas is pumped across the first transverse bore by means of a mechanical pump via a liquid-nitrogen cooled sorption trap containing Linde 5A molecular sieve. The pressure in this pumping stage is approximately $1 \mathrm{~Pa}$. The sorption trap was provided with a heater for regenerating the sieve at $300^{\circ} \mathrm{C}$. For the two-stage version. a second differential pumping unit with a liquid-nitrogen trapped oil diffusion pump followed, operating at a pressure of less than $5 \times 10^{-4} \mathrm{~Pa}$.

It has been demonstrated (Lancaster et al 1978) that three stages of differential pumping are sufficient to reach a pressure of some $10^{-8} \mathrm{~Pa}$ in an ultrahigh vacuum chamber during lamp operation.

(viii) The adjustable mount can be seen in figure 3. The lamp stands on three conical bearings and is held by means of a tension spring. The vertical axis of rotation runs through the rear bearing. Rotation around the axis is facilitated by adjusting screw 1, which moves the push rod and the sliding carriage. Screws 2 and 3 serve to lift or lower the front or rear bearing by means of two levers. The screws can be adjusted when the lamp is operating by use of rotatable high-vacuum manipulator.

\subsection{Spatial light-beam profiles}

The knowledge of the spatial light-beam profile is important in angle-resolved photoionisation experiments, because it determines size and shape of the effective target. The beam was scanned by a photon detector in a distance of $0.67 \mathrm{~m}$ behind the end of the light capillary. A double ion chamber of the type described by Samson (1964) was used as absolute photon detector: photoion currents which are produced when the light beam passes subsequently through two gas cells of identical lengths were simultaneously measured with two electrometers (Keithley). The great advantage of the double ion chamber is that it is self-calibrating in the sense that neither the exact

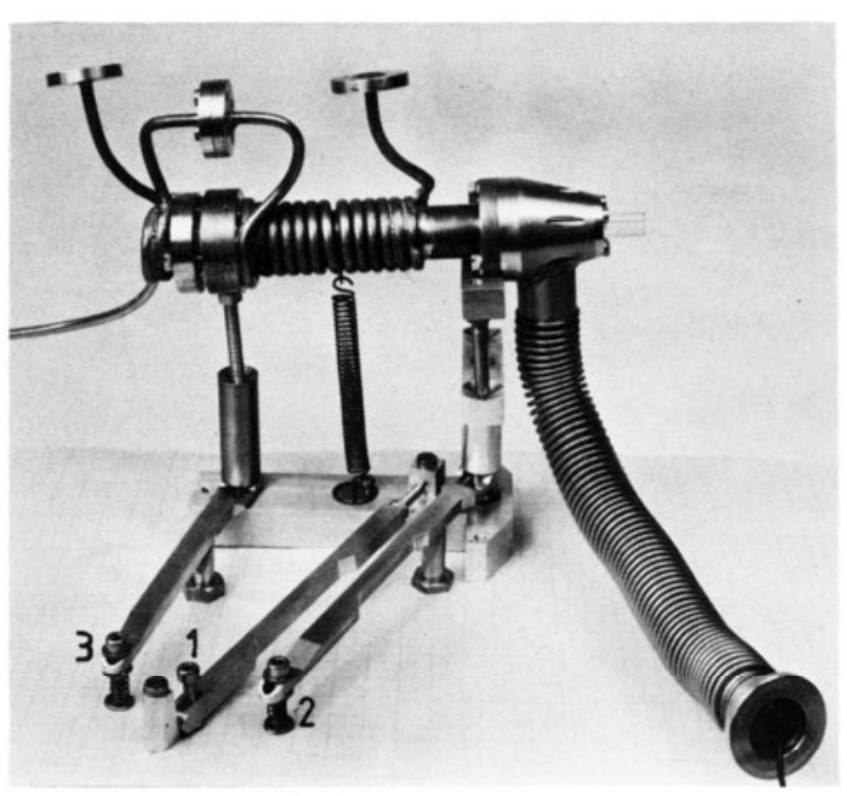

Figure 3. Photograph of the lamp and its adjustable mount. See text for screws $1,2,3$.

absolute pressure nor the absolute cross section of the absorbing gas in the chamber needs to be known. The entrance aperture of the detector had the dimension $1 \mathrm{~mm} \times 1 \mathrm{~mm}$ so that the measurement yielded directly the photon flux per $\mathrm{mm}^{2}$ in a distance of $0.67 \mathrm{~m}$ behind the lamp.

The experimental results of the scan are shown in figure 4. The photon flux was measured as a function of the position of the aperture perpendicular to the light-beam axis. The profile for Hel light was scanned using argon in the ion chamber and running the lamp at a high pressure, so that almost no Hell radiation was created in the discharge (see $\$ 2.4$ ). When

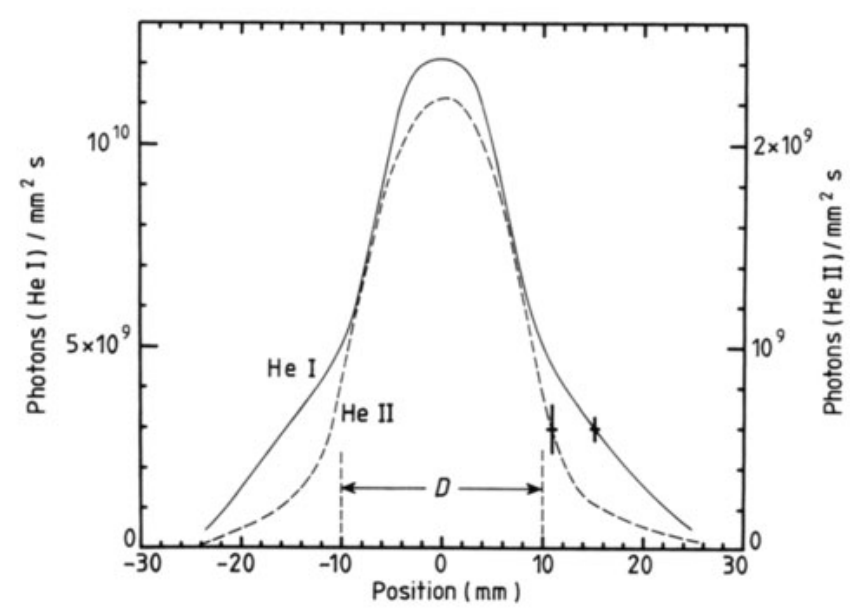

Figure 4. Spatial beam profiles for Hel (left ordinate) and HeII (right ordinate) resonance radiation. The profiles have been scanned in a distance of $0.67 \mathrm{~m}$ to the lamp. The abscissa is the distance from the beam axis. See text for $D$. The absolute photon fluxes (ordinates) have not been obtained under optimum discharge conditions with respect to the number of photons emitted. 
recording the HeII curve, the ion chamber was filled with helium, which cannot be ionised by the intense $\mathrm{HeI}$ lines.

The horizontal error bars show the width of the entrance aperture, the vertical error bars represent the uncertainty in measurements of the small photoion currents (sometimes below $10^{-11} \mathrm{~A}$ ). Diameter $D$ follows from a simple geometrical consideration assuming that photons are neither scattered at inner walls of the light capillary or gas atoms, nor absorbed and re-emitted by gas emerging from the lamp. $D$ reflects an angular divergence of $\pm 15 \mathrm{mrad}$. The widths of the measured beam profiles at half maximum show good agreement with the 'purely geometrical' diameter $D$. The tails of the curves show, however, that a non-negligible amount of radiation is scattered or reemitted in the lamp, particularly for the radiation with longer wavelength. In the experiments this zone of scattered light around the central beam of 'direct' light was partly screened by a circular aperture in order to have a well-defined beam diameter.

\subsection{Absolute photon intensities}

The total photon flux through the target region $(30 \mathrm{~mm}$ behind the end of the light capillary) was determined by integrating beam profiles which were recorded as described in $\S 2.2$. It is worth noting that the same intensity values have been obtained within the error bars by using a gold-coated photocathode as a detector (Samson 1967). Relative intensities of different resonance lines in the undispersed wavelength mixture were estimated from corresponding peak intensities in photoelectron spectra, taking into account the well-known photoionisation cross sections. The relative intensities agree quite well with those found by Weiss (1977) and Coatsworth et al (1978). All measurements were made under optimum discharge conditions and after baking the system for some hours.

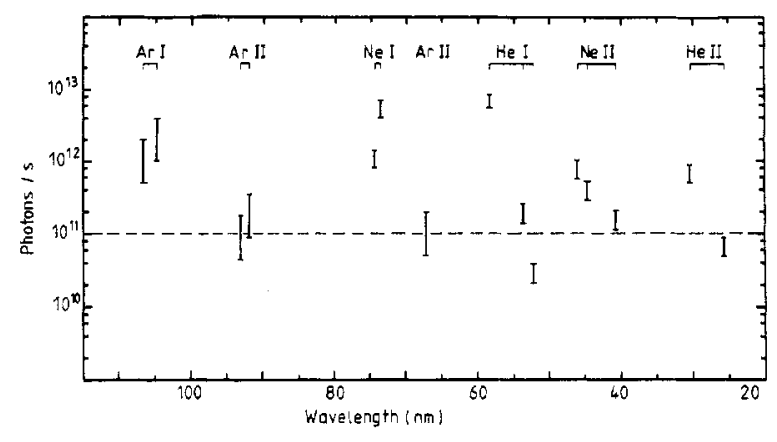

Figure 5. Photon intensities of the strongest rare-gas resonance lines. The error bars represent the total photon flux through the target region in a beam of $2.5 \mathrm{~mm}$ diameter, achieved with a $2 \mathrm{~mm}$ ID light capillary. The broken line indicates the order of magnitude in photon intensities, above which angular-resolved spin polarisation measurements with photoelectrons from gaseous targets become feasible.

The absolute photon intensities are shown in figure 5. On top of the figure the designations of the lines are given (I, atomic line; II, line of the singly-charged ion). The intensity values were measured when a light capillary of $2 \mathrm{~mm}$ inner diameter was used. If the diameter was $0.8 \mathrm{~mm}$, the photon intensities were reduced by a factor of 6 , which is just the ratio of the corresponding sectional areas.

In literature one finds several absolute photon intensities of $\mathrm{HeI}$ and HeII radiation obtained with resonance lamps without monochromator. Table 1 shows a list of the photon fluxes

Table 1. Comparison of HeI and HeII photon fluxes reported in literature. tot: photon flux of the total light beam (beam diameter is not stated by these authors). Present values: HeI, $8 \times 10^{12} \mathrm{~s}^{-1} ; \mathrm{HeII}, 7 \times 10^{11} \mathrm{~s}^{-1}$.

\begin{tabular}{|c|c|c|c|}
\hline \multirow[b]{2}{*}{ Type of lamp } & \multicolumn{2}{|c|}{ Photon flux } & \multirow[b]{2}{*}{ Reference } \\
\hline & HeI & HeII & \\
\hline $\begin{array}{l}\text { Windowed microwave } \\
\text { discharge }\end{array}$ & $6 \times 10^{10} \mathrm{~mm}^{-2} \mathrm{~s}^{-1}$ & 一 & Kinsinger et al (1972) \\
\hline $\begin{array}{l}\text { Windowless microwave } \\
\text { discharge }\end{array}$ & $10^{10}-10^{11} \mathrm{~mm}^{-2} \mathrm{~s}^{-1}$ & $5 \times 10^{7}-5 \times 10^{8} \mathrm{~mm}^{-2} \mathrm{~s}^{-1}$ & Rowe et al (1973) \\
\hline $\begin{array}{l}\text { Windowless hollow } \\
\text { cathode discharge }\end{array}$ & $10^{10} \mathrm{~mm}^{-2} \mathrm{~s}^{-1}$ & - & Dehmer and Berkowitz (1974) \\
\hline $\begin{array}{l}\text { Windowed glow } \\
\text { discharge }\end{array}$ & $10^{12} \mathrm{~s}^{-1}$ (tot) & $10^{11} \mathrm{~s}^{-1}$ (tot) & Fellner-Feldegg et al (1974) \\
\hline $\begin{array}{l}\text { Windowless microwave } \\
\text { discharge in } \\
\text { magnetic field }\end{array}$ & $3-5 \times 10^{11} \mathrm{~s}^{-1}($ tot $)$ & some $10^{10} \mathrm{~s}^{-1}(\mathrm{tot})$ & Vorburger et al (1976) \\
\hline $\begin{array}{l}\text { Windowless microwave } \\
\text { discharge }\end{array}$ & $10^{8} \mathrm{~mm}^{-2} \mathrm{~s}^{-1}$ & $\left.3 \times 10^{5} \mathrm{~mm}^{-2} \mathrm{~s}^{-1}\right)$ & \\
\hline $\begin{array}{l}\text { Windowless glow } \\
\text { discharge }\end{array}$ & $5 \times 10^{8} \mathrm{~mm}^{-2} \mathrm{~s}^{-1}$ & $3 \times 10^{6} \mathrm{~mm}^{-2} \mathrm{~s}^{-1}$ & Bruck et al (1976) \\
\hline $\begin{array}{l}\text { Low pressure } \\
\text { arc }\end{array}$ & $10^{9} \mathrm{~mm}^{-2} \mathrm{~s}^{-1}$ & - & \\
\hline $\begin{array}{l}\text { Windowless } \\
\text { duoplasmatron }\end{array}$ & $3 \times 10^{10} \mathrm{~mm}^{-2} \mathrm{~s}^{-1}$ & $2 \times 10^{7} \mathrm{~mm}^{-2} \mathrm{~s}^{-1}$ & \\
\hline $\begin{array}{l}\text { Windowless glow } \\
\text { discharge }\end{array}$ & $1.8 \times 10^{11} \mathrm{~s}^{-1}($ tot $)$ & - & Mason et al (1977) \\
\hline $\begin{array}{l}\text { Windowed microwave } \\
\text { discharge }\end{array}$ & $10^{10}-10^{11} \mathrm{~s}^{-1}(\mathrm{tot})$ & - & Pellach and Sar-El (1978) \\
\hline $\begin{array}{l}\text { Windowless glow } \\
\text { discharge }\end{array}$ & $1.6 \times 10^{12} \mathrm{~mm}^{-2} \mathrm{~s}^{-1}$ & $1.4 \times 10^{11} \mathrm{~mm}^{-2} \mathrm{~s}^{-1}$ & This work \\
\hline
\end{tabular}



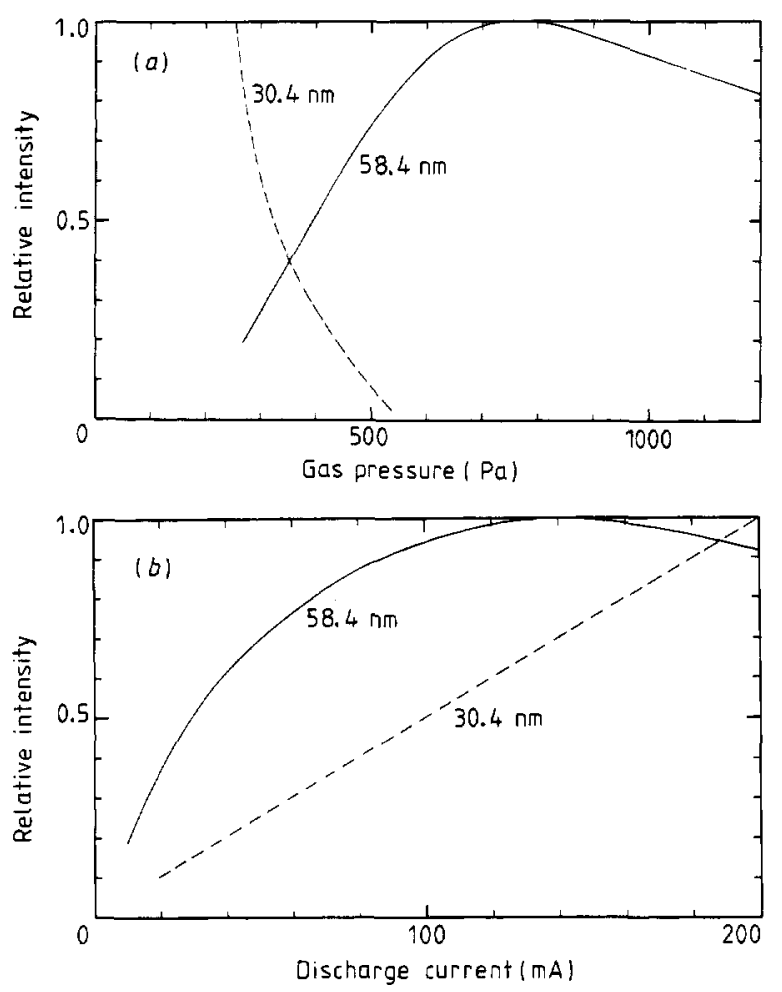

Figure 6. Photon intensity (relative curves) of the HeI $(58.43 \mathrm{~nm})$ and the HeII $(30.38 \mathrm{~nm})$ line as a function of the helium pressure $(a)$ and of the discharge current $(b)$. The abscissa in $(a)$ represents the reading of the gauge (figure 2), which is higher than the pressure in the discharge tube.

obtained by different groups. It should be kept in mind that for the values of figure $6(a)$. The $\mathrm{HeI}$ intensity shows a maximum at approximately $800 \mathrm{~Pa}$ and decreases towards lower pressures, whereas the HeIl line appears only below $600 \mathrm{~Pa}$ and increases sharply with decreasing pressure until the discharge breaks off at approximately $200 \mathrm{~Pa}$, depending on the cleanness of the helium. Similar curves have been observed for neon and argon. This behaviour agrees qualitatively with the results of other authors (Samson 1967, Poole et al 1974, Ueno et al 1977. Coatsworth et al 1978). It should be mentioned that, according to figure 5 , the absolute ordinates in figure $6(a)$ and $(b)$ for HeI and HeII are different.

(ii) The light intensity as a function of the discharge current is plotted in figure $6(b)$. The HeI intensity saturates above $100 \mathrm{~mA}$ and even seems to decrease at higher currents. In contrast to that, the HeII intensity increases almost linearly with the discharge current. The power supply of the lamp was voltageand current-stabilised $(0-6 \mathrm{kV}, 0-200 \mathrm{~mA})$. The high voltage was sufficient to start the discharge. To maintain a stable discharge, a $2 \mathrm{k} \Omega$ ballast resistor in series with the power supply was used. After ignition of the lamp, the voltage between cathode and anode drops to approximately $600 \mathrm{~V}$ for helium ( $450 \mathrm{~V}$ for neon), nearly independently of the discharge current. Only at very low pressures (i.e. good Hell or NeII conditions) the voltage at the discharge can rise up to more than $1000 \mathrm{~V}$.

(iii) Particularly the HeII and Nell intensities depend most sensitively on the cleanness of the lamp and the gas system. After each change of discharge gas, necessarily connected with aeration of the first valve, the inlet system was baked at about $200^{\circ} \mathrm{C}$ and the lamp at $100^{\circ} \mathrm{C}$ for some hours. During this time boiling water was led through the cooling pipes. In normal operation the lamp was also baked before each period of HeII or
NeIl production. When running the lamp in the HeII mode, the highest thermal power is produced (up to $200 \mathrm{~W}$ ) and the pressure of the discharge gas is as low as possible (see figures $6(a)$ and $(b)$ ). Thus high concentrations of impurities ejected from cathode or discharge capillary can accumulate. To avoid this, the Hell mode was interrupted whenever possible and the discharge region was flushed by clean gas under higher pressure.

\section{Practical performance in angular-resolved photoelectron spectroscopy}

Started by the fundamental theoretical work of Cooper and Zare (1969) and Buckingham et al (1970), the angular distribution of photoelectrons has received a continuously increasing interest on both the theoretical and the experimental side. According to theory, the differential cross section for photoionisation with unpolarised light (pure dipole transitions and randomly oriented target atoms or molecules presupposed) is given by the expression

$$
\frac{\mathrm{d} \sigma}{\mathrm{d} \Omega}(\theta)=\frac{Q}{4 \pi}\left[1-\frac{\beta}{2} P_{2}(\cos \theta)\right] .
$$

Here $Q$ is the total photoionisation cross section, $\theta$ is the angle between the incoming photon and outgoing electron momentum and $P_{2}(\cos \theta)=\frac{3}{2} \cos ^{2} \theta-\frac{1}{2}$. The asymmetry parameter $\beta$ is a measure for the deviation of the angular distribution from an isotropic one $(\beta=0)$ and does not exceed the values

$$
\beta=2\left(\text { i.e. } \frac{\mathrm{d} \sigma}{\mathrm{d} \Omega} \propto \sin ^{2} \theta\right)
$$

and

$$
\beta=-1\left(\text { i.e. } \frac{\mathrm{d} \sigma}{\mathrm{d} \Omega} \propto \cos ^{2} \theta\right) .
$$

$\beta$ contains important information about photoionisation dynamics, because it is a function of the transition matrix elements and phaseshift differences of continuum wavefunctions.

\subsection{Experimental arrangement}

The photoemission efficiency (number of photoelectrons per incoming photon) of solid surfaces reaches several percents in the vuv (Samson 1967). Therefore even a very small fraction of the high photon flux grazing a solid wall near the target region can cause an electron background that exceeds the true signal. Our photoionisation chamber, shown in figure 7, with the targetbeam nozzle and the aperture system for the light beam, was designed particularly for a high photoelectron signal with a minimum of background electrons. To achieve this, the light beam diameter is well defined by means of two apertures, the first of which is kept at a voltage of $+40 \mathrm{~V}$ in order to draw off photoelectrons emitted from the rims of the apertures. After crossing the target beam, the vLV-light intensity is monitored by a gold-coated photocathode which is tilted against the light beam so that no reflected photons can enter the ionisation chamber. The electrons ejected from the photocathode (which is grounded via a nanoammeter) are collected by a small electrode at a positive voltage.

The target beam is produced by a nozzle with a single bore of diameter $0.8 \mathrm{~mm}$ and length $14 \mathrm{~mm}$. In the present arrangement this nozzle proved to be advantageous as compared with a multi-capillary array; for equal gas throughput it yielded considerably higher photoelectron count rates and also the diameter of the gas beam was smaller than using the (nonfocusing) array. An adjusting screw allows the nozzle to be positioned to within a few tenths of a $\mathrm{mm}$ below the light beam. Hence the ionisation volume (spatial section of light and target 


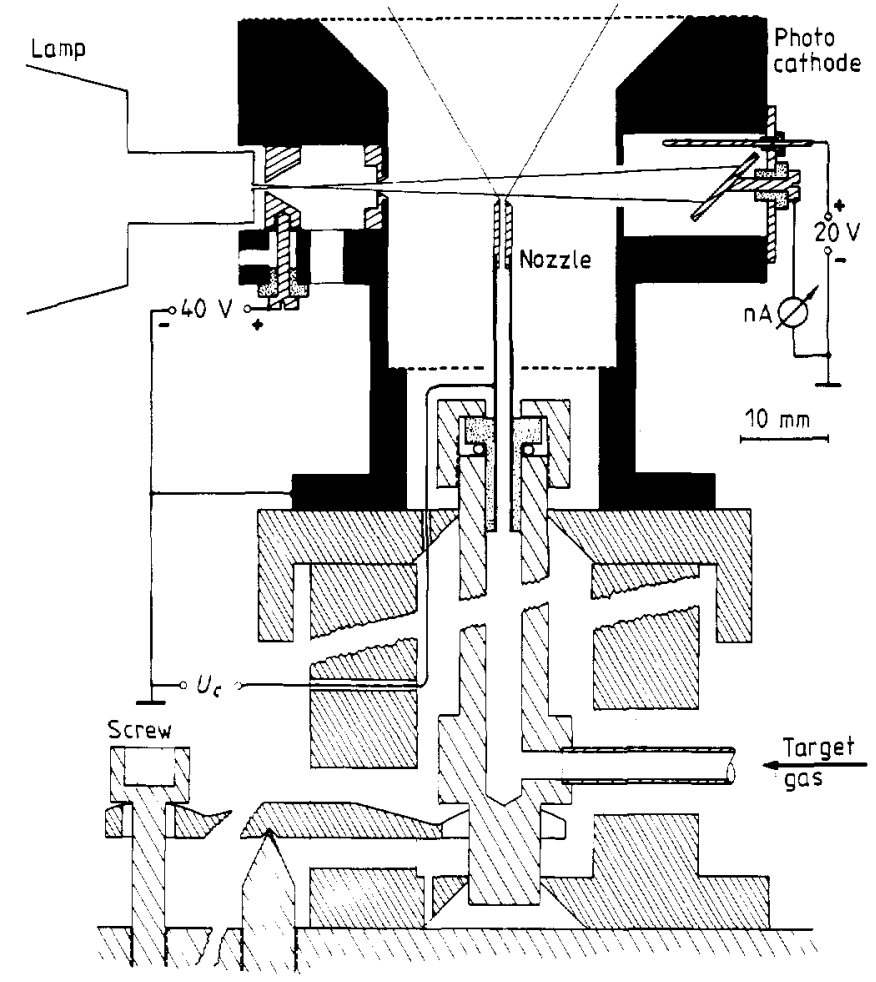

Figure 7. Vertical section of the photoionisation chamber. Materials: black, copper; hatched, aluminium; dotted, insulators; dashed, shielding grids.

The height of the nozzle can be adjusted by the screw. The entrance aperture of the electron spectrometer lies outside the drawing plane.

beam) can be kept small, an important requirement in angular distribution studies.

The target region has to be free of disturbing magnetic and electric fields, which would deflect the electron trajectories and thus falsify the angular distribution. The earth's magnetic field has been compensated to less than $1 \%$ by means of three pairs of Helmholtz coils. The ionisation chamber was coated with graphite and two fine grids (molybdenum mesh $0.25 \mathrm{~mm}$; wire $0.025 \mathrm{~mm}$ ) served as an electrical shield. Due to contact voltages the nozzle can have a different potential than the inner walls of the ionisation chamber. The resulting electric field above the nozzle gives rise to a poorly-defined zero of energy. This manifests itself in a deteriorated energy resolution. Such contact potentials were compensated by applying a small correction voltage $U_{\mathrm{c}}$ between nozzle and ionisation chamber. The voltage was determined empirically by minimising the peak widths in the photoelectron spectra.

Figure 8 shows the whole set-up used for the measurements of the asymmetry parameter $\beta$. The photoelectrons are ejected from the target atoms or molecules by photons from lamp 1 or lamp 2, mounted under fixed angles. The electron spectrometer is a cylindrical mirror analyser with outer and inner radii of 90 and $35 \mathrm{~mm}$, respectively, and a focusing distance of $246 \mathrm{~mm}$. The (virtual) entrance slit is the phototarget itself (approximate size $2.5 \mathrm{~mm}$ ) and the width of the exit slit can be varied between 0.5 and $3 \mathrm{~mm}$. Fringe field corrections are made by means of graphitised Mycroy plates. The angular acceptance is $\pm 5^{\circ} 30^{\prime}$. For pass energies less than $4 \mathrm{eV}$ the resolution has been measured to be better than $30 \mathrm{meV}$ FWHM. After passing the spectrometer, the photoelectrons are detected by the channeltron multiplier $\mathrm{C} 1$ connected with the usual counting electronics. In case a liquid helium cryopump $\left(50001 \mathrm{~s}^{-1}\right.$ pumping speed) was used to trap the target beam, count rates of up to $10^{6}$ electrons $\mathrm{s}^{-1}$ have been measured in the $\mathrm{Ar}^{-}\left({ }^{2} \mathrm{P}_{3 / 2}\right)$ peak at the $\mathrm{HeI}(58.43 \mathrm{~nm})$ wavelength. Background count rates (target beam switched off) were less than $50 \mathrm{~s}^{-1}$. During measurements of photoelectron polarisation, Cl was replaced by an accelerator for $120 \mathrm{keV}$ and a Mott-scattering detector. The second channeltron multiplier $\mathrm{C} 2$ serves as reference detector without energy-dispersing element.

\subsection{Experimental method}

The angles between the axes of lamp 1 and lamp 2 and the direction of observation are the 'magic' angle $\theta_{\mathrm{m}}=54^{\circ} 44^{\prime}$ ' and

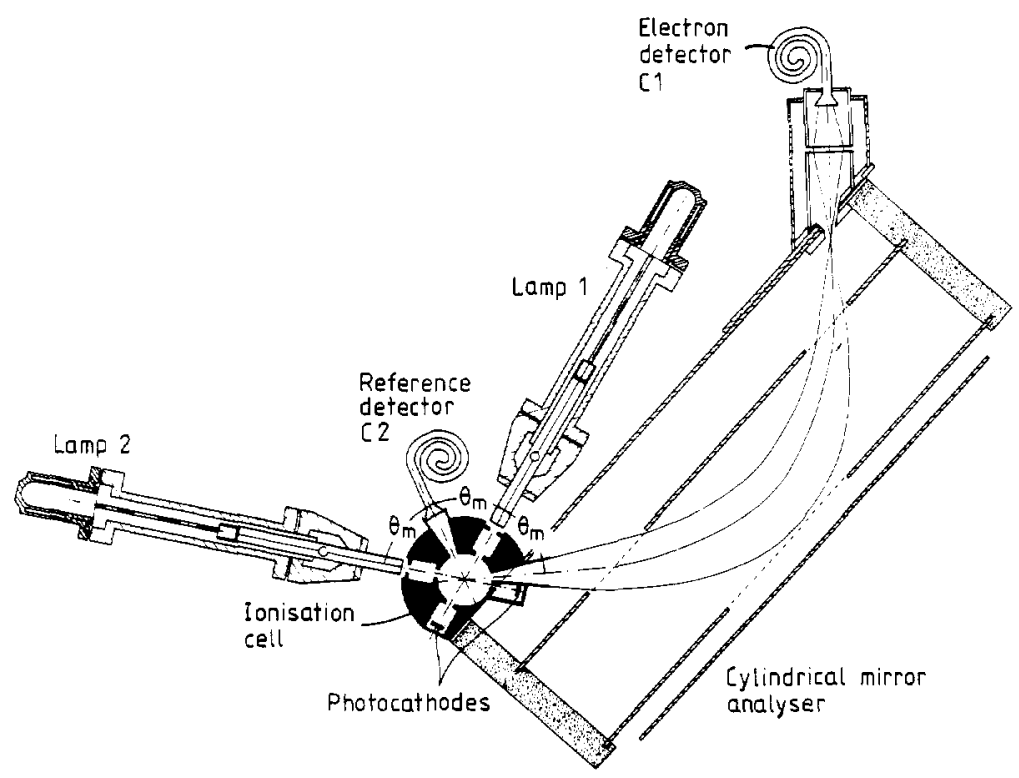

Figure 8. Horizontal section of the experimental arrangement for the angular-distribution measurements. $\theta_{\mathrm{m}}$ is the magic angle $54^{\circ} 44^{\prime}$. 
$3 \theta_{\mathrm{m}}$, respectively. With $P_{2}\left(\cos \theta_{\mathrm{m}}\right)=0$ and $P_{2}\left(\cos 3 \theta_{\mathrm{m}}\right)=0.889$ equation (1) yields the ratio of the corresponding photoelectron intensities

$$
I\left(3 \theta_{\mathrm{m}}\right) / I\left(\theta_{\mathrm{m}}\right)=1-0.444 \beta \text {. }
$$

This relation, however, cannot be applied directly to intensities measured by $\mathrm{C} 1$. First, the electron intensities must be normalised to equal photon intensities. This can be done using the signal in the reference detector $\mathrm{C} 2$, which is mounted under $\theta_{\mathrm{m}}$ with respect to both lamps 1 and 2 . Second, the solid angle interval accepted by the spectrometer has to be taken into account. A numerical estimation showed that the solid-angle correction is negligible as compared with the statistical errors and the error connected with the following point. Third, a correction due to the extended target region is necessary. This is a non-negligible contribution caused by the fact that the intersection volume of the light beam with the cone accepted by the spectrometer depends on the angle $\theta$ between the two directions. Since the volume is proportional to $(\sin \theta)^{-1}$, the corresponding correction of the measured intensities is often referred to as 'sine correction' (Niehaus and Ruf 1972). The full sine correction, however, only holds if the target-gas pressure is constant over the whole intersection volume. But in the present arrangement the width of the target beam lies between only 2 and $3 \mathrm{~mm}$, because of the small distance between nozzle and light beam. Thus a calibration factor $\alpha$ must be introduced when the asymmetry parameter $\beta$ is determined from the experimental intensities $I_{\exp }\left(3 \theta_{\mathrm{m}}\right)$ and $I_{\exp }\left(\theta_{\mathrm{m}}\right)$. From equation (2) one obtains

$$
\beta=2.25\left(1-\frac{I\left(3 \theta_{\mathrm{m}}\right)}{I\left(\theta_{\mathrm{m}}\right)}\right)=2.25\left(1-\alpha \frac{I_{\exp }\left(3 \theta_{\mathrm{m}}\right)}{I_{\exp }\left(\theta_{\mathrm{m}}\right)}\right) .
$$

$I_{\exp }\left(3 \theta_{\mathrm{m}}\right)$ and $I_{\exp }\left(\theta_{\mathrm{m}}\right)$ were determined by numerical integration of the corresponding peak areas in photoelectron spectra recorded with a multichannel analyser during operation of lamp 2 or lamp 1, respectively. The electron background was subtracted and the intensities were normalised to an equal number of counts in the reference detector $\mathrm{C} 2$.

The factor $\alpha$ was determined with argon as calibration gas. utilising the results of an 'absolute' measurement of $\beta$ using linearly polarised radiation (Schönhense 1980). The calibration yielded $\alpha=0.83 \pm 0.03$. It has been shown that the correction factor is the same for several different permanent gases and that $\alpha$ is in good approximation also obtained theoretically for the present geometry, if a Lorentzian target-beam profile is assumed (Schönhense 1978). Comparison of the empirical value with the full sine correction $\left(\alpha_{s}=0.296\right)$ shows that the target gas is very far from being continuously distributed over the ionisation volume.

The uncertainty of the present measurement results mainly from the uncertainty in $\alpha$. On the other hand, $\alpha$ is the same for all $\beta$ parameters of a certain target. Hence the observation of relative differences in $\beta$ is limited by counting statistics only. For example, the small difference in $\beta$ for the two spin-orbit components in the valence-shell ionisation of argon (final ionic states ${ }^{2} \mathrm{P}_{3 / 2}$ and ${ }^{2} \mathrm{P}_{1 / 2}$ separated by $180 \mathrm{meV}$ ) was clearly observed. This sensitivity for small variations in $\beta$ is important for the discussion of the following section.

\subsection{Results obtained for $\mathrm{N}_{2}$ molecules}

Asymmetry parameters $\beta$ for photoionisation of $\mathrm{N}_{2}$ molecules were measured. The following reactions were investigated: $\mathrm{N}_{2}\left(\mathrm{X}^{1} \Sigma_{\mathrm{g}}^{+}\right)+h v(21.22 \mathrm{eV}) \rightarrow \mathrm{N}_{2}^{+}\left(\mathrm{X}^{2} \Sigma_{\mathrm{g}}^{+} v^{\prime}=0,1\right),\left(\mathrm{A}^{2} \Pi_{\mathrm{u}} v^{\prime}=0\right.$, $1,2,3),\left(\mathrm{B}^{2} \Sigma_{\mathrm{u}}^{+} v^{\prime}=0\right)+\mathrm{e}^{-}$. In figure 9 the results are plotted as a function of the photoelectron kinetic energy. The lower part of the figure shows the photoelectron spectrum at the magic angle (the spectrum has not been corrected for the transmission of the electron analyser). The full circles in the upper part are the

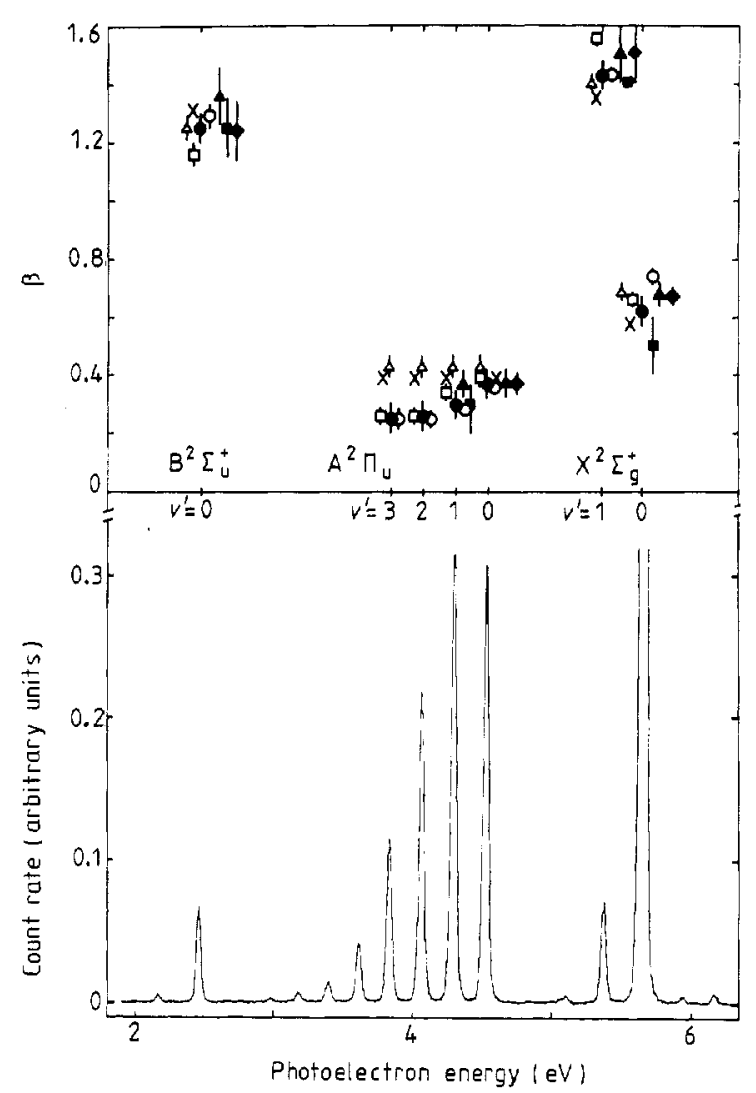

Figure 9. Lower part, photoelectron spectrum of $\mathrm{N}_{2}$ excited with $\mathrm{HeI}$ radiation. Upper part, corresponding asymmetry parameters $\beta$. Symbols: 0 , this work; $\bigcirc$, Mintz and Kuppermann (1978); $\diamond$, Katsumata et al (1979a); $\mathbf{0}$, Carlson and Jonas (1971); $\Delta$, Hancock and Samson (1976); $\triangle$, Niehaus and Ruf (1972); $\times$, Kibel et al (1979); $\square$, Kreile and Schweig (1980).

present results for $\beta$. For comparison we included $\beta$ values at $58.43 \mathrm{~nm}$ available in the literature. The classifications of the three different final ionic states are given in the figure. Also the final vibrational quantum numbers $v^{\prime}$ are denoted. In the graphic representation some of the experimental points of different authors had to be plotted at slightly different energies; the points collected in a group correspond to the same electron energy given by the corresponding peak in the lower part of the figure.

The agreement between all results of different authors is quite good. Particularly for the $\mathrm{A}^{2} \Pi_{u}$ band the present values agree excellently with those of Mintz and Kuppermann (1978) and Kreile and Schweig (1980). The variation in the angular distribution for different $v^{\prime}$ of this band was first observed by Carlson (1971). The first two members of the $\mathrm{X}^{2} \Sigma_{\mathrm{g}}^{+}$band have anomalously different angular distributions, which was observed by all authors. This anomalous behaviour has not yet been fully explained theoretically, whereas the variation of $\beta$ across the vibrational progression $\mathrm{A}^{2} \Pi_{u}\left(v^{\prime}=0,1,2,3\right)$ can be explained by variation of $\beta$ with photoelectron kinetic energy (Mintz and Kuppermann 1978). Angular distribution studies for the above reactions have also been carried out using monochromated synchrotron radiation (Marr et al 1979, Holmes and Marr 1980). The results of these authors at wavelengths close to $58.43 \mathrm{~nm}$ agree well with the present values.

\section{Conclusions}

A rare-gas resonance lamp has been built and tested which 
produces vuv-photon fluxes up to the order of $10^{13}$ photons s $^{-1}$ (HeI, NeI) in a beam of diameter $2.5 \mathrm{~mm}$. This high intensity made the first angularly and energetically resolved photoelectron spin polarisation studies feasable. The spatial profile of the light beam as well as the absolute photon intensities of several $\mathrm{He}, \mathrm{Ne}$ and $\mathrm{Ar}$ resonance lines have been measured. For twelve different wavelengths the lamp produces more than $10^{11}$ photons $\mathrm{s}^{-1}$. The intensity was optimised chiefly by three constructional features, namely (i) the windowless design, facilitated by means of differential pumping, (ii) the close distance between discharge capillary and photoionisation target, realised by placing the lamp inside the vacuum chamber, and (iii) the clean discharge conditions, achieved by building lamp and gas inlet system with UHV components only. Comparison with photon intensities reported in literature for various types of line sources shows that no higher photon fluxes have been obtained so far.

As an example for applications of the lamp, an angular distribution measurement with photoelectrons from $\mathrm{N}_{2}$ molecules is described and results for the asymmetry parameter $\beta$ are compared with a number of literature values reported for the same wavelength $(58.43 \mathrm{~nm})$. The good agreement in general but particularly concerning a slight variation of $\beta$ across the vibrational progression $\mathrm{N}_{2}^{+} \mathrm{A}^{2} \Pi_{\mathrm{u}}\left(v^{\prime}=0,1,2,3\right)$ demonstrates the efficiency of the experimental method employed.

\section{Acknowledgments}

It is a pleasure to thank Professor J Kessler (University of Münster) for his stimulating interest in the development of the lamp. Thanks are also due to B Willerding for her technical assistance in measuring the absolute intensities.

This work was supported by the Deutsche Forschungsgemeinschaft.

\section{References}

Bruck M, Bruckmüller R and Weissmann W 1976 Comparison of various types of vacuum ultraviolet light sources for photoelectron spectroscopy

4th Int. Conf, on Gas Discharges, Swansea, Wales (London: IEE) pp 407-10

Brundle C R, Roberts M W, Latham D and Yates K 1974 An ultrahigh vacuum electron spectrometer for surface studies J. Electron Spectrosc. Relat. Phenom. 3 241-61

Buckingham A D, Orr B J and Sichel J M 1970 Angular distribution and intensity in molecular photoelectron spectroscopy

Phil. Trans. R. Soc. A 268 147-57

Burger F and Maier J P 1979 'Charged particle oscillator' HeII photon source

J. Electron Spectrosc. Relat. Phenom. 16 471-4

Carlson T A 1971 Angular dependence of vibrational structure in the photoelectron spectra of $\mathrm{N}_{2}$ and $\mathrm{O}_{2}$ Chem. Phys. Lett. $923-6$

Carlson T A and Jonas A E 1971 Angular distribution of the photoelectron spectra for $\mathrm{Ar}, \mathrm{Kr}, \mathrm{Xe}, \mathrm{H}_{2}, \mathrm{~N}_{2}$ and $\mathrm{CO}$ J. Chem. Phys. 55 4913-24

Coatsworth L L, Bancroft G M, Creber D K, Lazier R J D and Jacobs P W M 1978 Charged particle oscillator and hollow cathode HelI lamps for high resolution photoelectron studies J. Electron Spectrosc. Relat. Phenom. 13 395-401

Cooper J and Zare R N 1969 Photoelectron angular distributions, in Lectures in Theoretical Physics, Vol. 11c, ed W E Brittin et al (New York: Gordon and Breach) pp $317-37$
Dehmer J L and Berkowitz J 1974 Partial photoionization cross sections for $\mathrm{Hg}$ between 600 and $250 \AA$. Effect of spin-orbit coupling on the ${ }^{2} \mathrm{D}_{5 / 2} /{ }^{2} \mathrm{D}_{3 / 2}$ branching ratio of $\mathrm{Hg}$ Phys. Rev. A 10 484-90

Fehsenfeld F C, Evenson K M and Broida H P 1965 Microwave discharge cavities operating at $2450 \mathrm{MHz}$

Rev. Sci. Instrum. 36 294-8

Fellner-Feldegg H, Gelius U, Wannberg B, Nilsson A G, Basilier E and Siegbahn K 1974 New developments in ESCA-instrumentation

$J$. Electron Spectrosc. Relat. Phenom. 5 643-89, and private communication

Hancock W H and Samson J A R 1976 Angular distribution of photoelectrons at $584 \AA$ using polarized radiation

J. Electron Spectrosc. Relat. Phenom. $9211-6$

Heinzmann U 1980 Spin polarized photoelectrons from atoms and molecules

Appl. Opt. $194087-91$

Heinzmann U, Schönhense $G$ and Kessler J 1980 Wavelength dependence of polarisation of photoelectrons ejected by unpolarised vUV radiation from argon and krypton atoms J. Phys. B: Atom. Molec. Phys. 13 L153-6

Holmes R M and Marr G V 1980 The angular distribution of photoelectrons from $\mathrm{N}_{2}, \mathrm{O}_{2}$, and $\mathrm{CO}$ as a function of photon energy

J. Phys. B: Atom. Molec. Phys. $13945-50$

Katsumata S, Achiba Y and Kimura K 1979a Photoelectron angular distribution of simple molecules at $30.4 \mathrm{~nm}$ photons J. Electron Spectrosc. Relat. Phenom. 17 229-36

Katsumata S, Nomoto K, Ohmori K, Kirihata Y, Yamazaki T, Achiba Y and Kimura K 1979b A simple HeII resonance lamp for use in UV photoelectron spectroscopy

J. Electron Spectrosc. Relat. Phenom. $16485-8$

Kibel M H, Leng F J and Nyberg G L 1979 Angular-

distribution $\mathrm{HeI} / \mathrm{NeI}$ photoelectron spectra of $\mathrm{H}_{2}, \mathrm{~N}_{2}$ and other small molecules

$J$ Electron Spectrosc. Relat. Phenom. 15 281-6

Kinsinger J A. Stebbings W L, Valenzi R A and Taylor J W 1972 Spectral evaluation of a sealed helium discharge lamp for studies in photoelectron spectroscopy

Anal. Chem. 44 773-7

Kreile J and Schweig A 1980 Photoelectron asymmetry parameters of $\mathrm{Ar}, \mathrm{Kr}, \mathrm{Xe}, \mathrm{H}_{2}, \mathrm{~N}_{2}, \mathrm{O}_{2}, \mathrm{CO}$ and $\mathrm{CO}_{2}$ : new measurements and a reconsideration of literature data J. Electron Spectrosc. Relat. Phenom. 20 191-211

Lancaster G M, Taylor J A, Ignatiev A and Rabalais J W 1978 Vacuum ultraviolet resonance line radiation source from rare gas atoms and ions for UHV photoelectron spectroscopy J. Electron Spectrosc. Relat. Phenom. 14 143-53

Marr G V, Morton J M, Holmes R M and McCoy D G 1979 Angular distribution of photoelectrons from free molecules of $\mathrm{N}_{2}$ and $\mathrm{CO}$ as a function of photon energy J. Phys. B: Atom. Molec. Phys. 12 43-52

Mason D C, Mintz D M and Kuppermann A 1977 Variable angle photoelectron spectrometer

Rev. Sci. Instrum. 48 926-33

Mintz D M and Kuppermann A 1978 Energy dependence of the differential photoelectron cross sections of molecular nitrogen J. Chem. Phys. 69 3953-66

Neddermeyer H, Heimann P und Roloff H F 1976 Ein hochauflösendes UV-Photoelektronenspektrometer mit CAMAC-Steuerung

J. Phys. E: Sci. Instrum. 9 756-62 
Newburgh R G, Heroux L and Hinteregger H E 1962 Two light sources for use in the extreme ultraviolet Appl. Opt. 1 733-7

Niehaus A and Ruf M W 1972 The variation of photoelectron angular distributions with energy

Z. Phys. 252 84-94

Pellach E and Sar-El H Z 1978 Vacuum UV spectrum of helium lamp with tubings of different diameters

J. Electron Spectrosc. Relat. Phenom. 14 259-66

Poole R T, Liesegang J, Leckey R C G and Jenkin J G 1974 A high intensity uv source for photoelectron spectroscopy J. Electron Spectrosc. Relat. Phenom. 5 773-82

Rosebury F 1965 Handbook of Electron Tube and Vacuum Techniques (Reading, Massachusetts: Addison-Wesley) p 152

Rowe J E, Christman S B and Chaban E E 1973 Simple. windowless lamp for ultrahigh vacuum photoemission spectroscopy

Rev. Sci. Instrum. 44 1675-6

Samson J A R 1964 Absolute intensity measurements in the vacuum ultraviolet J. Opt. Soc. Am. 54 6-15

Samson J A R 1967 Techniques of Vacuum Vltraviolet Spectroscopy (New York: Wiley)

Samson J A R and Liebl H 1962 Duoplasmatron as a vacuum ultraviolet light source

Rev. Sci. Instrum. 33 1340-3

Schönhense G 1978 Bau von Resonanzlampen zur Erzeugung intensitätsreicher vuv-Strahlung und deren Verwendung bei der Messung der Winkelverteilung von Photoelektronen aus Permanentgasen

Diploma Thesis, University of Münster

Schönhense G 1980 Angular dependence of the polarization of photoelectrons ejected by plane-polarized radiation from argon and xenon atoms

\section{Phys. Rev. Lett. 44 640-3}

Schönhense G, Heinzmann U, Kessler J and Cherepkov N A 1982 b Photoelectron polarization in $\mathrm{Hg} 6 \mathrm{~s}^{2}$ subshell-ionization with unpolarized light: new aspect of the Fano effect

Phys. Rev. Lett. 48 603-6

Schönhense G, Schäfers F, Heinzmann U and Kessler J 1982a Angle- and spin-resolved photoelectron spectroscopy of the $\mathrm{Hg} 5 \mathrm{~d}^{10}$ subshell Z. Phys. A-Atoms and Nuclei 304 31-40

Ueno N, Ikegami A, Hayasi Y and Kiyono S 1977 Intensity dependence of the HeII resonance radiation on helium gas pressure Japan J. Appl. Phys. 16 1655-8

Vorburger T V, Waclawski B J and Sandstrom D R 1976 Improved microwave-discharge source for uv photoemission Rev. Sci. Instrum. 47 501-4

Weiss M J 1977 'Impurity' lines in the helium resonance source for photoelectron spectroscopy

J. Phys. E: Sci. Instrum. $1023-5$

Wuilleumier F J 1981 Atomic physics with synchrotron radiation: past, present and future; A tomic Physics Vol. 7 pp. 491-527 ed. Daniel Kleppner and Francis M Pipkin (New York: Plenum) 